\title{
Seguridad del uso de inhibidores de la bomba de protones en el embarazo
}

Safety of using proton-pump inhibitors in pregnancy

\section{Objetivos}

Estudiar la relación entre la exposición a los inhibidores de la bomba de protones (IBP) en las fases tempranas del embarazo, y el desarrollo de malformaciones importantes en el niño.

\section{Diseño}

Estudio poblacional de cohortes.

\section{Lugar}

Dinamarca.

\section{Pacientes}

Se identificaron todos los niños nacidos en el país entre 1996 y 2008 a través de los registros médicos respectivos. Se calculó el inicio de la gestación restando a la fecha de nacimiento la edad gestacional.

\section{Evaluación de los factores de riesgo}

A partir de los datos del registro nacional de prescripciones se identificaron las madres a las que se les había prescripto un IBP desde las cuatro semanas antes de la fecha de inicio de la gestación hasta el momento del parto. Se identificaron los casos de malformaciones mayores a partir de los registros clínicos, y se clasificaron según los criterios de la European Surveillance of Congenital Anomalies (EUROCAT).

Se recogieron datos sobre posibles factores de confusión: datos sociodemográficos, antecedentes maternos (edad, paridad, utilización de fármacos y hábitos tóxicos), y antecedentes del embarazo. No se tenían datos sobre la utilización materna de ácido fólico. Se dividió el tiempo de exposición en: cuatro semanas previas al embarazo, primer, segundo y tercer trimestre.

\section{Medición de resultados principales}

Odds ratio* de malformaciones congénitas en niños nacidos de
Pasternak B. y col. N Engl J Med 2010;363:2114-23.

madres expuestas a IBP comparado con aquellos nacidos de madres no expuestas.

\section{Resultados principales}

Se incluyeron en el estudio 840.968 nacimientos, de los cuales casi 35.000 fueron múltiples. Un $2,6 \%$ de los recién nacidos presentaron alguna malformación importante. La prescripción de IBP aumentó a lo largo del tiempo, acercándose al final del estudio al $2 \%$ de las gestaciones. El IBP más prescripto fue el omeprazol.

Un 3,4\% de los hijos de madres expuestas presentaron una malformación importante (según la clasificación de EUROCAT).

Tabla 1: Odds ratio ajustado de tener descendencia con malformaciones importantes en relación con la exposición a IBP y el tiempo de exposición.

\begin{tabular}{l|c|c|c} 
& $\begin{array}{c}\text { 4 semanas } \\
\text { previas }\end{array}$ & 1er. trimestre & $\begin{array}{c}\text { 2do. y } \\
\text { 3er.trimestre }\end{array}$ \\
\hline Cualquier IBP & $1,39(1,10$ a 1,76) & $1,10(0,91$ a 1,34$)$ & $1,09(0,92$ a 1,29) \\
\hline Omeprazol & $1,06(0,65$ a 1,73) & $1,05(0,79$ a 1,40) & $1,04(0,85$ a 1,26) \\
\hline Pantoprazol & $1,09(0,62$ a 1,92) & $1,33(0,85$ a 2,08) & $1,39(0,79$ a 2,44) \\
\hline Lanzoprazol & $1,91(1,30$ a 2,80) & $1,13(0,77$ a 1,67) & $1,55(0,97$ a 2,47) \\
\hline Rabeprazol & $1,97(0,44$ a 8,72) & $2,14(0,60$ a 7,68$)$ & $3,27(0,37$ a 29,12) \\
\hline Esomeprazol & $1,35(0,82$ a 2,23) & $1,19(0,77$ a 1,84) & $0,92(0,50$ a 1,70) \\
\hline
\end{tabular}

\section{Conclusiones}

El tratamiento con IBP en el primer trimestre del embarazo no se asoció en forma significativa a un mayor riesgo de malformaciones importantes. Se halló asociación significativa entre la utilización de lanzoprazol dentro de las cuatro semanas previas a la concepción y aumento de riesgo de malformaciones congénitas.

Fuente de financiamiento/conflicto de intereses de los autores: Danish Medical Research Council y la Lubeck Foundation.

\section{Comentario}

El reflujo gastroesfágico sintomático es frecuente en el embarazo y la mayor parte de las embarazadas lo presentan en algún momento del mismo desde el primer trimestre. Aunque contamos con medidas higiénico-dietéticas para buscar el alivio sintomático (evitar el tabaco y los alimentos ácidos que relajan el tono del esfínter esofágico inferior, elevación de la cabecera de la cama en caso de síntomas nocturnos), los IBP constituyen un tratamiento más eficaz para este trastorno.
En estudios individuales y en un metaanálisis previo no se han reportado indicios de que los IBP aumenten el riesgo de malformaciones congénitas ${ }^{1-2}$. El hecho de que el omeprazol sea el fármaco del grupo más prescripto e incluido en estos estudios hace que sea el que mayores garantías de seguridad ofrece, por lo que se ha ido incorporando progresivamente a las recomendaciones de tratamiento del reflujo en el embarazo.

Farm. Gerardo Foenquinos [ Farmacia del Plan de Salud del Hospital Italiano de Buenos Aires. gerardo.foenquinos@ hospitalitaliano.org.ar ]

Foenquinos G. Seguridad del uso de Inhibidores de la Bomba de Protones en el embarazo. Evid Act Pract Amb Ene-Mar 2012;15(1):19. Comentado de: Pasternak B, Hviid A. Use of Proton-Pump Inhibitors in Early Pregnancy and the Risk of Birth Defects. N Engl J Med 2010;363:2114-2123. PMID: 21105793.

Referencias bibliográficas

1. Diav-Citrin O, Arnon J, Shechtman S. y col. The safety of proton pump inhibitors in pregnancy: a multicentre prospective controlled study. Alimentary Pharmacology \& Therapeutics 2005; 21: 269-275

2. Nikfar S, Abdollahi M, Moretti ME, et all. Use of proton pump inhibitors during pregnancy and rates of major malformations: A meta-analysis. Dig Dis Sci 2002;47:1526-29. 